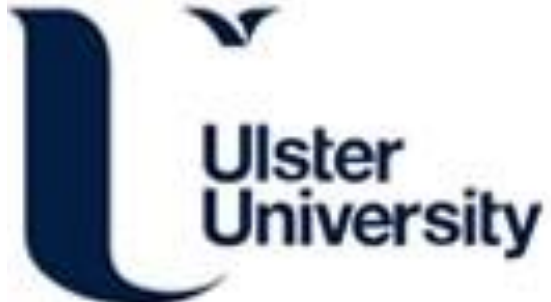

\section{Monte Carlo modeling and optimization of buffer gas positron traps}

MARJANOVIĆ, S., \& Petrović, Z. (2017). Monte Carlo modeling and optimization of buffer gas positron traps. Plasma Sources Science and Technology, 26, [024003 ]. https://doi.org/10.1088/1361-6595/aa5308

Link to publication record in Ulster University Research Portal

\section{Published in:}

Plasma Sources Science and Technology

Publication Status:

Published (in print/issue): 13/01/2017

DOI:

10.1088/1361-6595/aa5308

\section{Document Version}

Author Accepted version

\section{General rights}

Copyright for the publications made accessible via Ulster University's Research Portal is retained by the author(s) and / or other copyright owners and it is a condition of accessing these publications that users recognise and abide by the legal requirements associated with these rights.

\section{Take down policy}

The Research Portal is Ulster University's institutional repository that provides access to Ulster's research outputs. Every effort has been made to ensure that content in the Research Portal does not infringe any person's rights, or applicable UK laws. If you discover content in the Research Portal that you believe breaches copyright or violates any law, please contact pure-support@ulster.ac.uk. 


\title{
Monte Carlo modeling and optimization of buffer gas positron traps
}

\author{
Srđan Marjanović ${ }^{1}$ and Zoran Lj Petrovici ${ }^{1,2}$ \\ ${ }^{1}$ Institute of Physics, University of Belgrade, POB 6811080 Zemun Belgrade, \\ Serbia \\ ${ }^{2}$ Serbian Academy f Sciences and Arts, 11001 Belgrade, Serbia
}

E-mail: msrdjan@ipb.ac.rs

Abstract. Abstract ide ovde

\section{Introduction}

Positron beams have been readily available for use in experiments since the 1970's [1,2,3], but it is the discovery and development of buffer gas positron traps (or Penning Malmberg Surko-type positron traps) by Surko and coworkers $[4,5]$ that allowed the field of low energy positrons, and antimatter physics in general to advance at a faster pace [6]. Current state of the art devices, using solid neon moderators [7] and optimized trapping arrangements are capable of efficient trapping, storing, cooling and manipulation of the accumulated positrons, with $\sim 10^{8}-10^{9}$ positrons stored from seconds to several minutes [8,9]. Buffer gas positron traps have been successfully used in experiments to measure, with high accuracy, the scattering cross-sections for positron-matter interaction [10-13], create positronium (Ps) and Ps molecules [14], form antihydrogen atoms [15,16,17], perform Ps laser spectroscopy [18] or do materials science studies [19].

In principle positron traps filled by buffer gas belong to the physics of ionized gases. The positron ensemble is very non-equilibrium until the last moments of thermalization and it is all driven by fields and collisions with the background gas. The only difference to the low-temperature plasmas is lack of multiplication of the main species, positrons although secondary electrons are readily produced if conditions allow it. Nevertheless, this being the tool of atomic and molecular physics the analysis of such devices was mainly based on beam like models and considerations. While revealing most of the pertinent aspects of operation quantitative description requires full kinetic modeling/theory allowing us to follow actual energy distribution and assign importance to different processes according to their contribution. In that respect this paper aims to apply a procedure developed (and well tested/benchmarked) for swarms to model positron traps, their performance and optimization. Hopefully development of such a model may open roads to more new ideas that may be tested by modeling before building devices.

Swarm like models and experiments have been attempted for positrons in past. While measurements of transport coefficients proved to be too complicated and had little luck, measurements of swarm like properties [20] such as thermalization time [21,22] were numerous with a potential to yield 
similar averages that could be used to verify the completeness of the set of cross sections. There were specialized calculations of thermalization times [23] but also some considerations of the basic transport theory [24]. Recently the focus returned back to the swarm studies [25-29] discovering kinetic phenomena such as negative differential conductivity (NDC) for the bulk drift velocity with a complete lack of a similar effect for the flux drift velocity. This is all due to magnitude of the non-conservative Positronium (Ps) formation cross section and its shape [29]. The progress was enabled by the availability of the cross section data mainly thanks to the advancements in Surko traps [10-13]. Thus there seems to be a degree of poetic justice in attempting to use the data to improve the performance of the device that enabled us to obtain those data in the first place. Another line of research recently opened by the availability of the data is modeling of tracks, kinetics and positron induced chemistry in dense gases, liquids and hopefully models of the living tissues [30-34].

In principle, a buffer gas positron trap is a Penning Malmberg trap, consisting of cylindrical electrodes of increasing radius effectively creating three stages of gas pressure (figure 1). The highest pressure in the first stage, and the lowest pressure in the third stage are achieved by continuous gas feed and differential pumping [5]. Positrons are trapped inside the electrode structure by an inelastic collision in the first stage, with a suitably selected buffer gas. The length of the first stage and the gas pressure are selected to ensure et least one trapping collision per single pass of a positron through the trap. Through subsequent inelastic collisions the positrons are confined first to stage II and then finally to the stage III where gas pressure is the lowest (hence they can be stored for longer periods of time), and they thermalize to room temperature. An axial magnetic field is introduced to prevent transport in the radial direction.

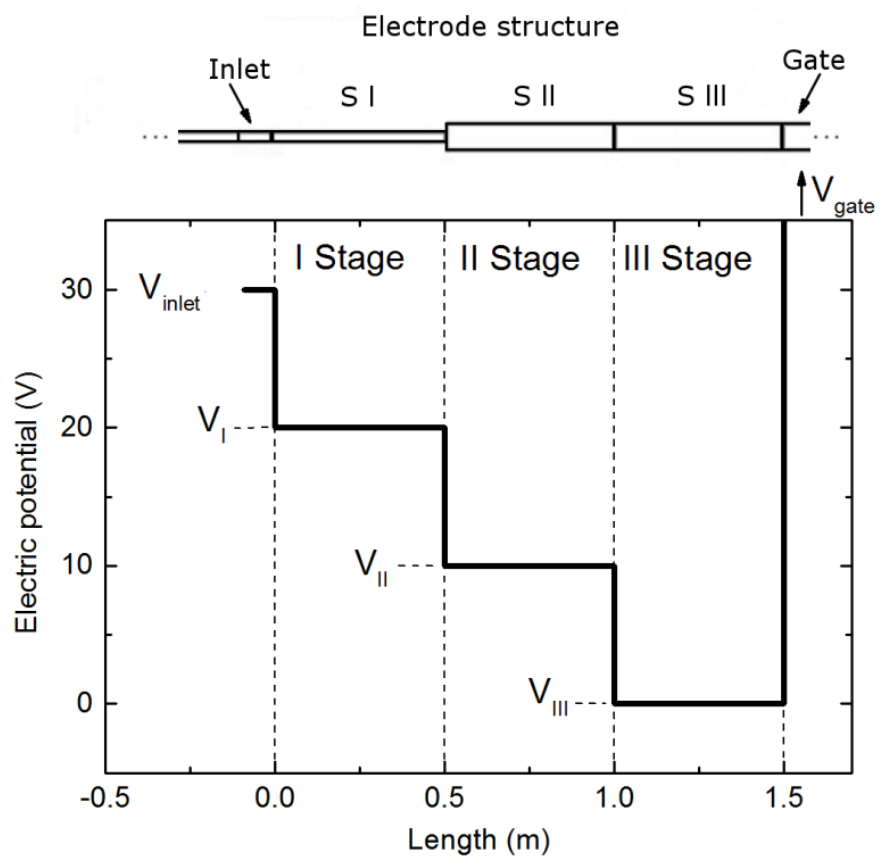

Figure 1. Schematic of the electrode structure and the axial electric potential profile of the buffer gas positron trap. The moderated positron beam enters from the left.

Although various gasses can achieve a certain degree of trapping, the most efficient gas in trapping positrons has proven to be $\mathrm{N}_{2}$ due to the conveniently positioned (in relation to the positronium formation cross-section) $a^{1} \Pi$ electron excitation cross-section [10], which competes efficiently with the main loss mechanism - Ps formation. The two processes are competing, with electron excitation being dominant in the 8.6 - $11.5 \mathrm{eV}$ energy range (see figure 2). We do not know of other transitions that show dominance over Ps formation with an equally large energy loss. This energy range presents a window in 
which trapping by using excitation of $\mathrm{N}_{2}$ is efficient. Usually, to reduce thermalization times, a gas with high vibrational excitation cross-section, like $\mathrm{CF}_{4}$ or $\mathrm{SF}_{6}$ is introduced in the third stage.

Depending on the intended purpose of the trapping device different design choices can be made to optimize a certain beam property or minimize construction cost. For instance, lately a number of buffer gas traps has been realized in the so called two stage variant where electrodes of only two different radii have been used which employes higher pressure in the accumulation region. Such a device provides shorter thermalization time, smaller device size and cheaper construction cost, but reduces positron lifetimes. Due to the reduced lifetime, accumulation times and, consequently, the total number of positrons per pulse are also reduced [35-38]. The final stage of the trap often utilizes an rotating electric field to compress positrons radially, reducing the output beam diameter and increasing the density of trapped positrons [35, 39, 40].

Three stage traps on the other hand have lower pressure in the accumulation region, enabling longer accumulation times and longer storage times. Thus it is possible to achieve high particle densities and formation of single component positron plasma in the accumulation region which can then be manipulated in the similar manner as the electron plasmas [41]. This enables construction of complex devices such as multicell traps [42] intended for storing $~ 10^{12}$ positrons for weeks.

This paper focuses on simulation of trapping and cooling of positrons in a buffer gas positron trap using a standard well established Monte Carlo technique in a computer code that is well tested for electron benchmarks [43-46], taking an in depth look at the loss processes that reduce trapping efficiency, optimization of gas pressure and electrode voltages for a fixed geometry as well as the evolution of positron energy distribution during thermalization inside the apparatus.

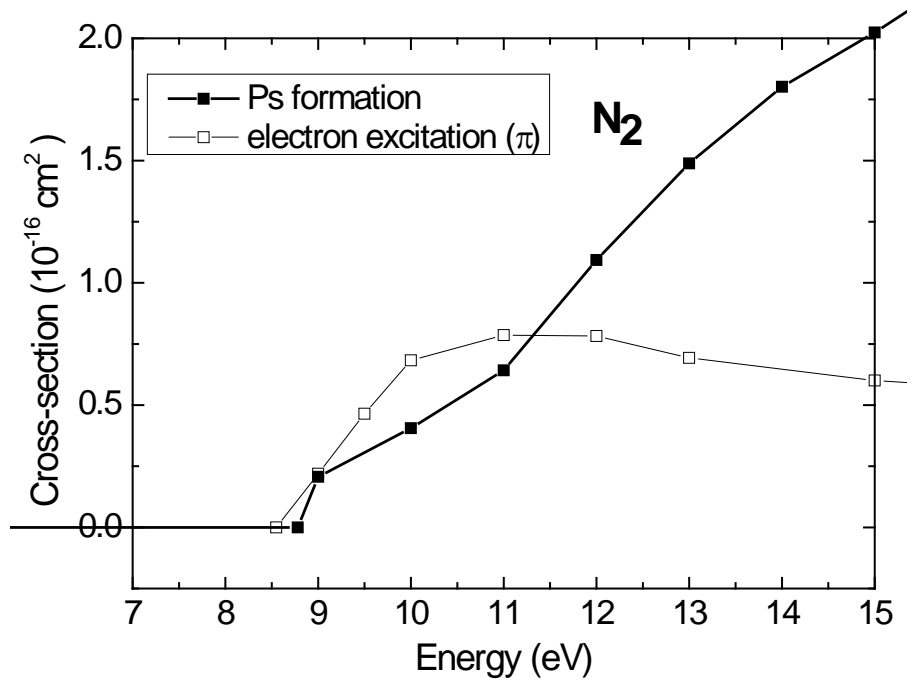

Figure 2. Measured cross-section values for a ${ }^{1} \Pi$ electron excitation (open squares) , and Ps formation (full squares)[10]

\section{Model}

A model of the gas filled Surko trap is formulated by separating the entire device into three cylindrical segments, each corresponding to a stage of the trap. For each segment we define dimensions (length and radius) as well as the pressure and composition of the background gas, applied magnetic field and electric potential of the corresponding electrode. Also defined are the inlet and outlet electrode potentials (as seen in figure 1). The inlet electrode potential serves to define the energy of positrons as they enter the trap, and 
also to define the potential barrier which prevents the trapped positrons from escaping the trap, back to the moderator. The outlet electrode potential remains constant, significantly higher than the maximum positron energy, and prevent positrons from exiting the trap. Within a particular stage (electrode) the simulation assumes no electric field, with positrons exhibiting cyclotron motion due to axial magnetic field. Positrons crossing from stage to stage are either accelerated/decelerated along the axis or reflected by the potential, depending on their energy, direction they are traveling and the potential difference between the corresponding electrodes. Positrons that reach the walls, or exit the trap along the axis, over the inlet electrode with enough axial energy to overcome the potential barrier are counted as lost.

The energy width of a positron beam obtained by a solid Ne moderator is 1.5 eV FWHM [7]. The simulated beam is assumed to have a triangular $1.5 \mathrm{eV}$ FWHM energy distribution with the velocity vector uniformly distributed in the $2 \pi$ sr space, with positrons going inside the trap $\left(\mathrm{v}_{\mathrm{z}}>0\right)$. Spatially, positrons are uniformly distributed inside $1.5 \mathrm{~mm}$ radius around the axis.

The simulation assumes pure $\mathrm{N}_{2}$ in stages I and II, and a $50 \%$ mixture of $\mathrm{N}_{2}$ and $\mathrm{CF}_{4}$ stage III. Cross-section sets for positron interaction with these molecules have been compiled previously [47] and have been used before in swarm simulations[22,25,48,49]. The differential scattering data is not available, therefore the simulation assumes isotropic scattering in the center of mass frame for all processes. These cross-sections have been used to describe positron interaction with the buffer gas through a standard Monte Carlo technique [50-52] which includes only binary collisions. Special care is taken to properly include thermal collisions [53] (gas is assumed to be at $300 \mathrm{~K}$ room temperature), and superelastic collisions through the principle of detailed balance. Positron trajectory between collisions is calculated analytically depending on the magnetic field strength. During trapping, positron density is low, therefore we are justified to neglect the space-charge effects.

The same simulation code and model has been used before in a preliminary presentation of positron trap operation [48] but more importantly, it has been used, and thoroughly tested in electron swarm modeling and related benchmark calculations [50,54]. Also it has proven viable in the so-called non hydrodynamic (non-local) development of the particle swarm. In such situations a full equilibrium between energy and momentum gained from the field and lost through collisions has not been reached. Under such conditions a number of kinetic phenomena develop for electrons [55] and also for positrons, so it is pertinent to transport of positrons in buffer gas traps.

\section{Results of simulations}

From the simulation, we are able to sample the temporal development of the number of positrons and mean energy in each segment, as well as the energy distribution of the entire swarm. We can also determine the number and type of loss processes in time as well as spatial distribution of positrons and other relevant parameters.

We first present a more general (non-optimized) trap setup, in order to investigate the main losses and see the scale of their influence on the trapping efficiency. Then, for the established trap geometry we perform the optimization, similarly to how it is done in experimental conditions [5], by first optimizing the pressure in stage I (trapping region), and then by optimizing the electrode potential to achieve best possible trapping efficiency.

\subsection{Generalized buffer gas design simulation}

Detailed parameters of the simulation are given in table 1. The main characteristic of the simulated trap is the fixed $10 \mathrm{~V}$ potential drop from the inlet electrode to the stage I electrode, then from stage I to stage II electrode and so on. This way, the energy of positrons inside stage I is $10.1 \mathrm{eV}$ (due to the source bias) added to the $1.5 \mathrm{eV}$ wide (FWHM) energy distribution. Electrode radius (20 mm) and pressure $\left(10^{-5}\right.$ Torr) in the third stage is more in line with the compact two stage designs. However we refer to it as the stage 
III to reflect the structure of electric potential. It can also be called the accumulation region. We should point out that, as the pressure within a stage is (assumed to be) constant, number of collisions within a segment corresponds to the product of gas pressure and the length of the stage, $p d=$ const. Therefore, we have selected the length of $0.5 \mathrm{~m}$ for all three stages, and the results, if needed, can be scaled for the arbitrary lengths or pressures. A preliminary presentation of the effect of the shapes of collision crosssections on the EDF (Energy distribution function) of positrons is given in [56]

Table 1 Simulation parameters for the generalized buffer gas trap

\begin{tabular}{llll}
\hline Parameters & Stage I & Stage II & Stage III \\
\hline Electrode radius (mm) & 5 & 20 & 20 \\
Length (m) & 0.5 & 0.5 & 0.5 \\
Pressure (Torr) & $10^{-3}$ & $10^{-4}$ & $10^{-5}$ \\
Background gas & $\mathrm{N}_{2}$ & $\mathrm{~N}_{2}$ & $\mathrm{~N}_{2}{ }^{0.5}+\mathrm{CF}_{4}{ }^{0.5}$ \\
Magnetic field (G) & 530 & 530 & 530 \\
Electrode potential (V) & 20 & 10 & 0 \\
\hline \multicolumn{2}{l}{ Initial conditions } \\
\hline Inlet electrode potential (V) & & 30 & \\
$\mathrm{e}^{+}$source bias (V) & 0.1 & \\
Energy distribution width (FWHM) (eV) & 1.5 & \\
\hline
\end{tabular}

The chosen stage to stage potential drop value of $10 \mathrm{~V}$ is certainly not optimal, in regards to total trapping efficiency. It was chosen, as a generic approach, to guarantee positrons have enough energy, within each stage, to excite electronic states of $\mathrm{N}_{2}$ molecule (while the cross-section for the Ps formation is not larger than excitation cross-section).

Results of simulation (for the parameters in table 1) are presented in figures 3 - 6. Figure 3 shows the evolution of the positron energy distribution functin (EDF) during thermalization. At each sampling point, energy distribution is normalized, so that the maximum value is equal to 1 , in order to achieve better readability across a wide energy range where magnitudes of the EDF vary by many orders of magnitude. Initially, positrons form a beam of $\sim 11.5 \mathrm{eV}$, with $1.5 \mathrm{eV}$ width. After some of the positrons lose $\sim 8.5 \mathrm{eV}$ and more in electron excitation of $\mathrm{N}_{2}$, they begin to form another broad peak at lower energies of few $\mathrm{eV}$. As both groups cross into stage II they are accelerated by $10 \mathrm{eV}$. Thus a narrower beam around $20 \mathrm{eV}$ forms as well as a group as the apparent continuation of the $10 \mathrm{eV}$ beam, but with a broader distribution. All the while some of the accelerated particles again experience electron excitation collisions again losing energy and forming the third group at around few $\mathrm{eV}$ and also replenishing the second group. As the bulk of the particles enters the third stage, the initial beam is differentiated into four groups with the remnants of the original unperturbed beam and groups of positrons that have undergone one, two or three electron excitation collisions. At a certain point in time ( $\left.t \sim 10^{-5} \mathrm{~s}\right)$, one can discern up to six different groups, some of them with very wide distributions. It is clear that these groups of positrons cannot be viewed as a beam especially at lower energies since their mean energy is of the same order of magnitude as their widths. The distribution is additionally spread through elastic and inelastic collisions. In the next stage higher energy groups dissipate forming eventually a broad distribution peaking at around 4-5 eV. This group's mean energy gradually decays during the period between around $1 \mathrm{~ms}$ and $20 \mathrm{~ms}$. Ultimately after $20 \mathrm{~ms}$ the entire swarm relaxes into a Maxwell-Boltzmann distribution, at room temperature, as expected. 


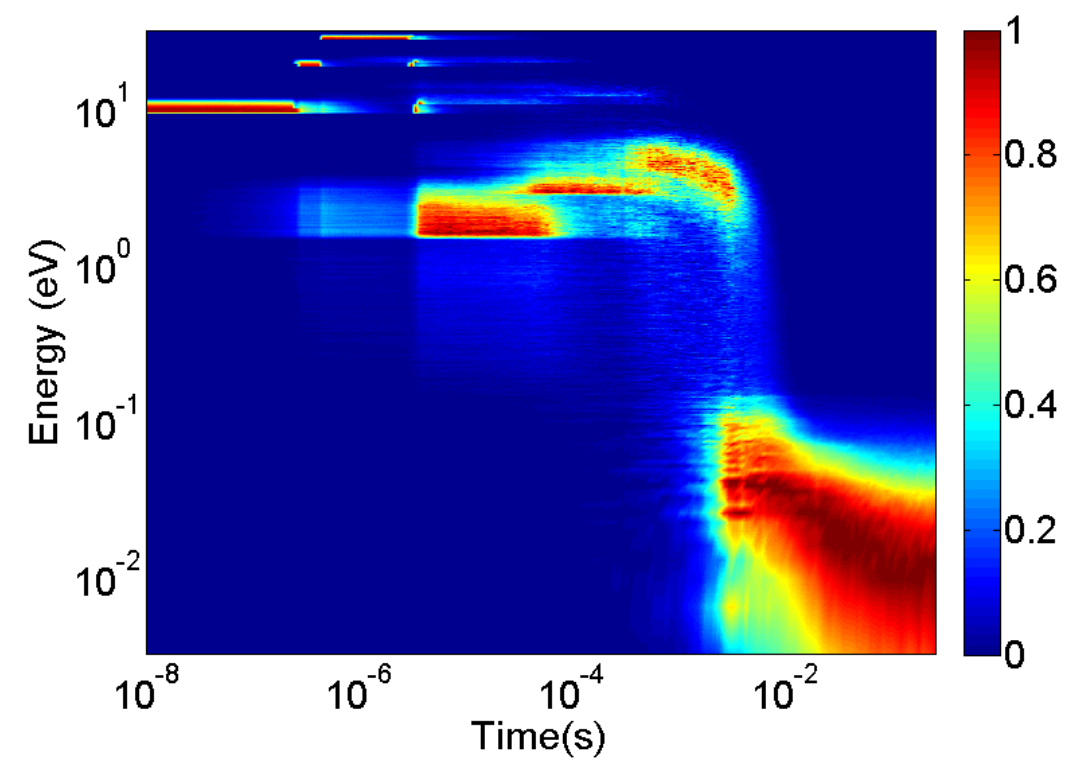

Figure 3. Evolution of positron energy distribution during thermalization inside a generalized buffer gas positron trap.

In Figure 4 we show the distribution function in the last stages of thermalization between mean energies of $\varepsilon=4.58 \mathrm{meV}(\mathrm{t}=1 \mathrm{~ms})$ and $\varepsilon=38.6 \mathrm{meV}(\mathrm{t}=250 \mathrm{~ms})$. In the first period the distribution is nothing like the Maxwellian of the same mean energy and only in the last stages (i.e. beyond $10 \mathrm{~ms}$ ) it converges. From figures such as those displayed here we may envisage which inelastic processes contribute mostly to the thermalization at different times. 

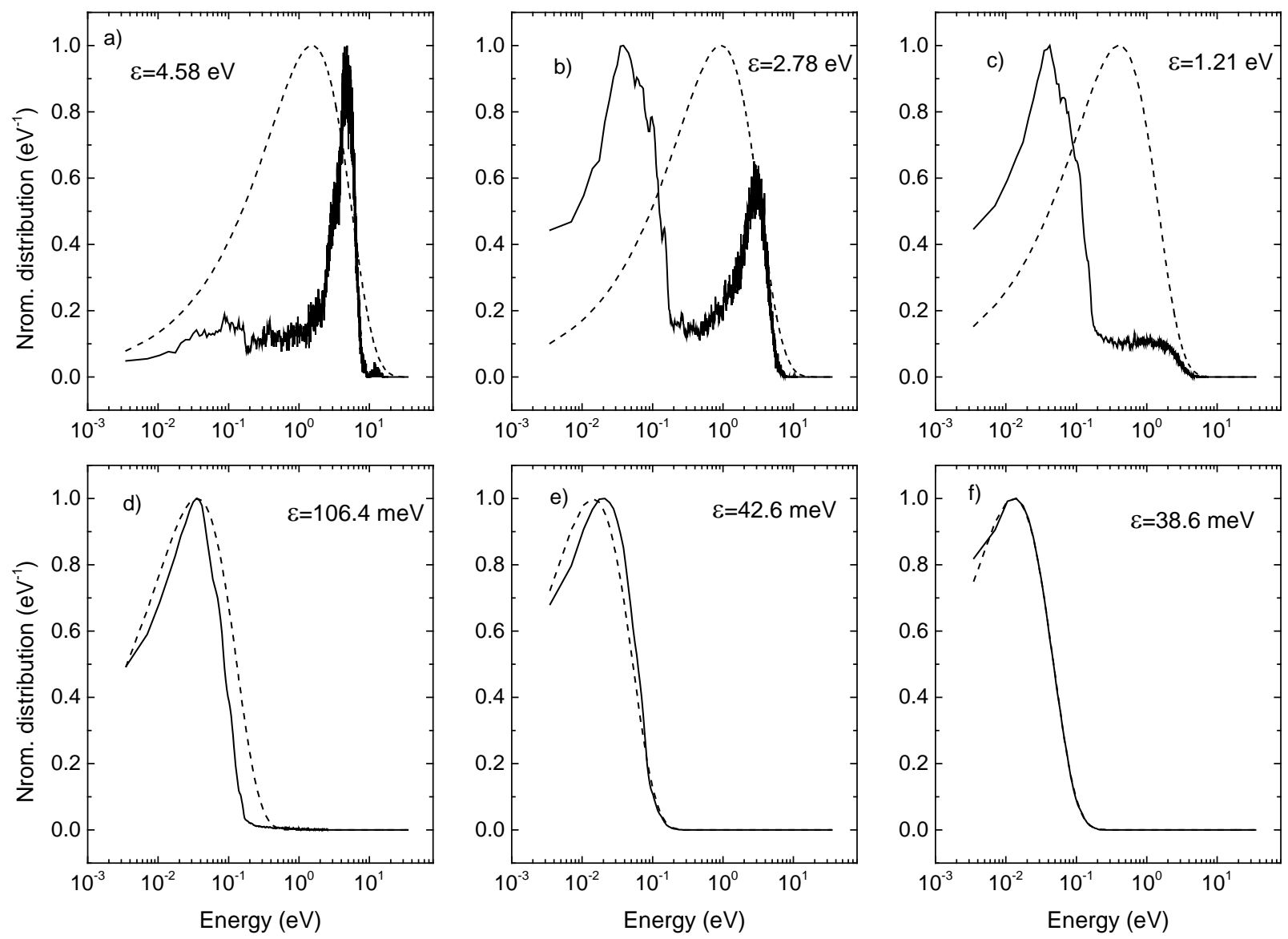

Figure 4. Evolution of positron energy distribution during the final stage of thermalization in accumulation region at moments a) $1.02 \mathrm{~ms}$, b) $3.52 \mathrm{~ms}$, c) $6.24 \mathrm{~ms}$, d) $11.05 \mathrm{~ms}$, e) $34.68 \mathrm{~ms}$, f) $256.62 \mathrm{~ms}$. Dashed lines represent MB distribution for the appropriate mean energy.

Figure 5 presents the number of positrons in individual stages during the thermalization inside the trap, normalized to the number of initial positrons at the beginning of the simulation. Positrons reach stage II after $\sim 0.2 \mu \mathrm{s}$, while traversing the entire trip, from the inlet to the outlet electrode and back, takes $\sim 3 \mu \mathrm{s}$. The time it takes for all of the positrons to leave the first and the second stage $\sim 0.1 \mathrm{~ms}$ and $\sim 1 \mathrm{~ms}$ respectively and that ratio corresponds to the ratio of the pressures in the stages. Ultimately, the number of trapped positrons accumulated in stage III is somewhat below 5\%.

It is clear that in real devices, the positrons do not enter the trap at the same time, but at the frequency of $\sim 10^{6} \mathrm{e}+/ \mathrm{s}$. This means that, with the exception of stage III, where the positrons are accumulated, at any point in time, there cannot be more than a few hundreds of positrons in a particular stage. Even in the third stage, which is capable of storing millions of positrons, with the exception of experiments that aim at creating positron plasmas, positron number density is well below the limit where the positron-positron interaction is relevant. This is another affirmation that swarm technique and Monte Carlo approach are suitable for describing the trapping process. 


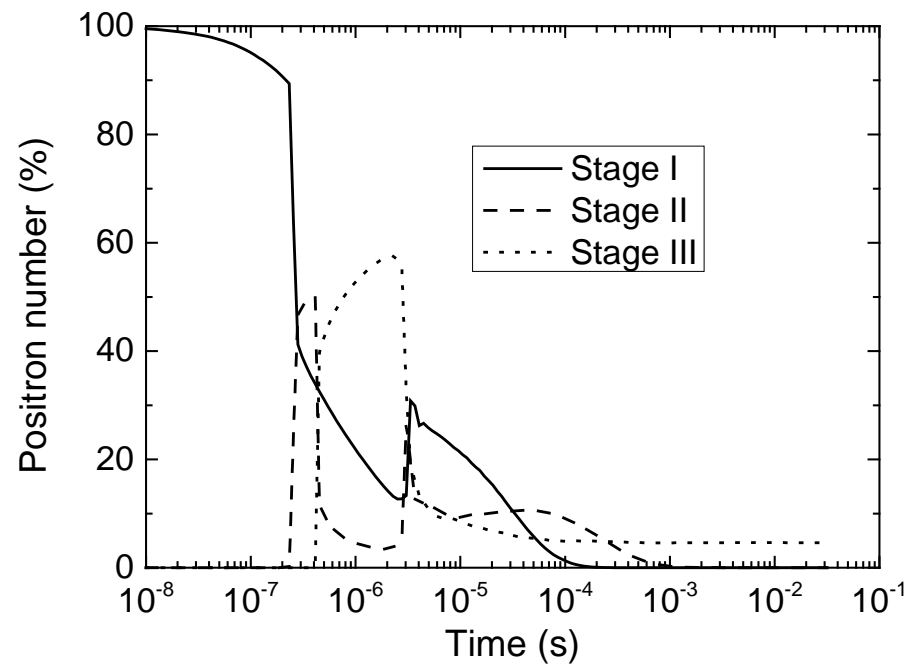

Figure 5. Relative number of positrons in individual stages over time.

Different processes that lead to loss of positron in the simulation are shown in figure 6 . The most prominent is the process of positronium formation (PsF), responsible for $\sim 80 \%$ of the losses. Positrons that bounce off the outlet electrode, and reach the inlet electrode with enough axial energy to overcome the potential barrier leave the device by traveling over the inlet electrode are labeled as escaped. Positrons that diffuse to the walls are removed from the simulation, as well as the positrons that annihilate directly. However, last two processes barely contribute to the losses on the simulated timescale, so in figure 6 their contribution is multiplied by a factor of 100 to make them visible.

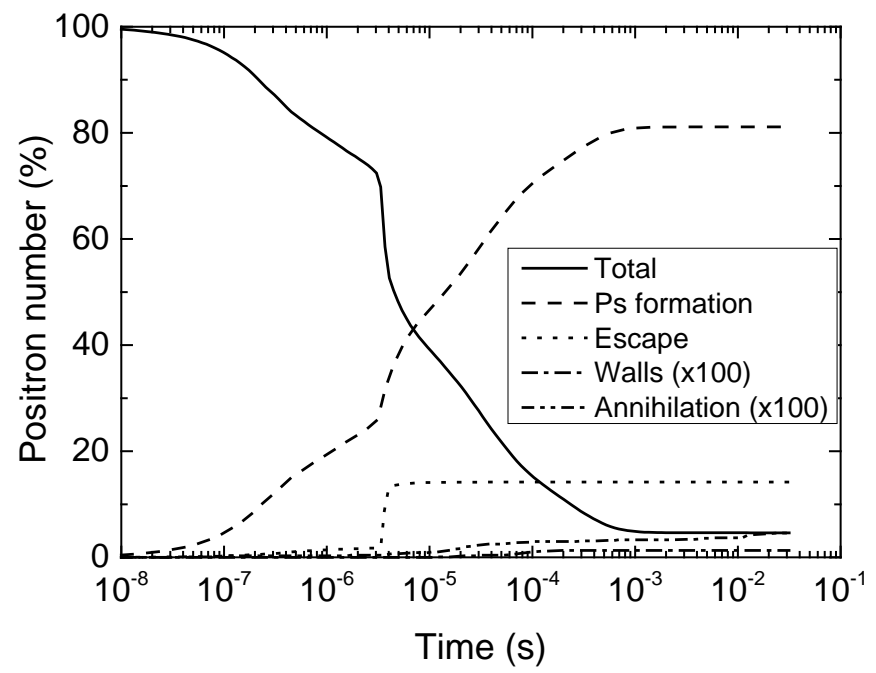

Figure 6. Total number of remaining positrons and the individual loss processes over time, normalized to the number of initialized positrons. Losses to walls and direct annihilation have been multiplied by a factor of 100 for visibility

Total efficiency of the simulated trap is $\sim 5 \%$. The largest contribution to positron loss is the Ps formation process which can be reduced by appropriately selecting electrode voltages, in order to better tune the positron energy distributions to the trapping window energies (figure 2). Increase of buffer gas pressure in the first stage does not change the ratio of Ps formation events, compared to the electron excitation collisions, but it does increase the probability that a positron interacts with a $\mathrm{N}_{2}$ molecule, reducing the number of escaped positrons. 
Figure 7 shows the evolution of radial distribution of positrons over time. Electrode radii used in existing devices, are determined by the pressures required for trap operation with regards to differential pumping. Apparently, those values are selected appropriately and diffusion to the walls does not contribute to positron losses in appreciable quantities, on the simulated timescale. With longer accumulation times, diffusion might lead to significant expansion of the swarm, but most devices currently employ rotating wall compression technique to reduce the positron cloud size and negate the effects of radial diffusion. Under the present circumstances the diffusion in the last stage, that is necessarily at the lowest pressure, increases the radius of the beam twofold.

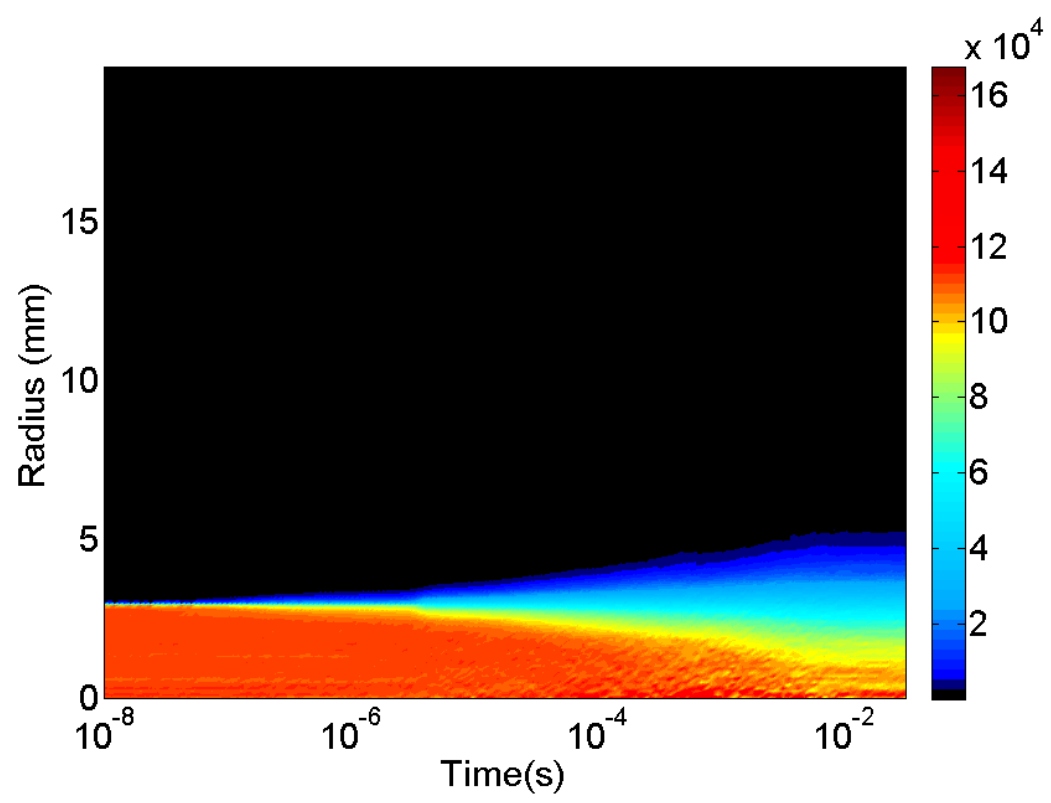

Figure 7. Evolution of radial distribution of the positron swarm during thermalization.

\subsection{Optimization of the buffer gas pressure in stage I}

Practical traps being used these days have properties close to those shown in table 1 as the foundation of our basic gas filled trap. All of the modifications, however small those may be, were done in a continuous empirical battle to optimize the trap further. The goal of this paper is to address one or two practical setups. In doing so, we shall address just which properties have been optimized and how to achieve the final choices and also try to elucidate which atomic and molecular processes play the key role in such development.

Optimization of the $\mathrm{N}_{2}$ pressure in the first stage may reduce the number of escaped positrons. Although different sources quote nitrogen pressure in the first stage as $\sim 10^{-3}$ Torr, it should be stated that it is difficult to exactly measure this value. Normally, experiments measure pressure in the third stage, and pressure profile in the rest of the trap is inferred from gas flow calculation and differential pumping. That is why the suggested value of 1 mTorr should be considered as an estimate. The actual optimal pressure in experiments is achieved by increasing the flow of nitrogen into the trap, until the number of positrons trapped per second saturates. A similar approach has been employed in the simulation.

A series of simulations have been performed using parameters from the table 1, while changing the gas pressure in the stage I between 0.5 mTorr and 9 mTorr. Results are shown in figure 8 . The saturation occurs for pressures above 3 mTorr. Under those conditions, all of the positrons that enter the trap either experience Ps formation or electron excitation in the first stage. A small number of positrons 
still escape the trap as they are backscattered by elastic collisions in the first stage, which is shown in subsection 3.4

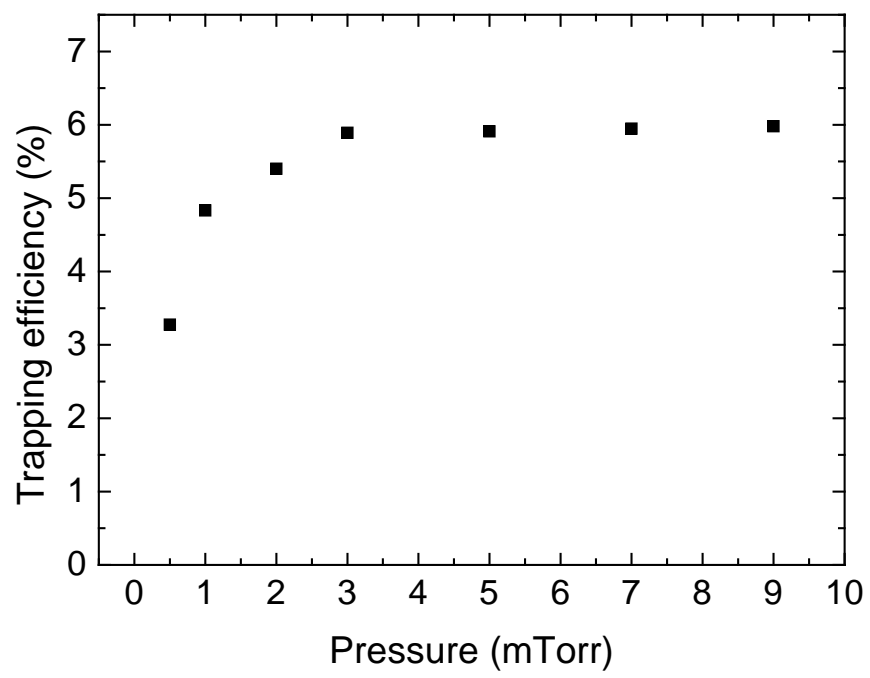

Figure 8. Simulated trapping efficiency as a function of $\mathrm{N}_{2}$ pressure in the stage I, for parameters given in table 1 .

\subsection{Optimization of electrode potentials}

Through optimization of electrode potentials we are able to improve the ratio of positrons that experience electron excitation collision versus those that are lost to Ps formation process, by tailoring the positron energy distribution. The way it is done in simulation is similar to how it was done in experiment [5].

First, we optimize the value of stage I electrode potential in respect to the potential of inlet electrode. Previously determined pressure of 3 mTorr is assumed for the $\mathrm{N}_{2}$ pressure in the first stage while the incoming beam properties (FWHM and source bias) are retained. Simulation is limited only to the first stage by setting the stage II electrode potential to a high value. A series of simulations are performed for different values of potential difference between the inlet and stage I electrode, with trapping efficiency defined as a portion of positrons retained inside the trap after all of the particles have either left the trap, or experienced Ps formation. Results of simulations are presented in figure 9. The optimal potential difference between the inlet and the stage I potential is $8.4 \mathrm{~V}$. For smaller values most positrons do not have enough energy to excite electron states of $\mathrm{N}_{2}$ molecule, while for the larger values positrons have too much energy and their energy distribution does not align with the trapping window, leading to more Ps formation. Achieved trapping efficiency in a single stage is $57 \%$ which is a direct consequence of the shape of cross-sections for $a^{1} \Pi$ electron excitation and Ps formation on one hand, and the width of energy distribution of positrons coming from the moderator on the other.

For optimization of stage II electrode, the electric potential of the inlet and stage I electrode have been set to provide, the previously determined, $8.4 \mathrm{~V}$ potential drop, while the stage III electrode potential has been set to a high value, effectively limiting the trap to two stages. Result of these simulation is show in figure 10. Optimal value for potential drop from stage I to stage II electrode is determined to be $8 \mathrm{~V}$ and the trapping efficiency achieved is $32 \%$. As we see, with every (optimized) potential drop, that is sufficiently deep to provide energy for another electron excitation of $\mathrm{N}_{2}$ we can expect $\sim 43 \%$ loss in number of positrons. Therefore, a three stage trap is expected to achieve at best an $\sim 18 \%$ trapping efficiency for the simulated incoming beam properties. Wider energy distribution of the incoming beam is likely to further reduce the maximum obtainable efficiency due to the limited width of the trapping window energy, shapes of the cross-sections and energy distribution of incoming positrons. 


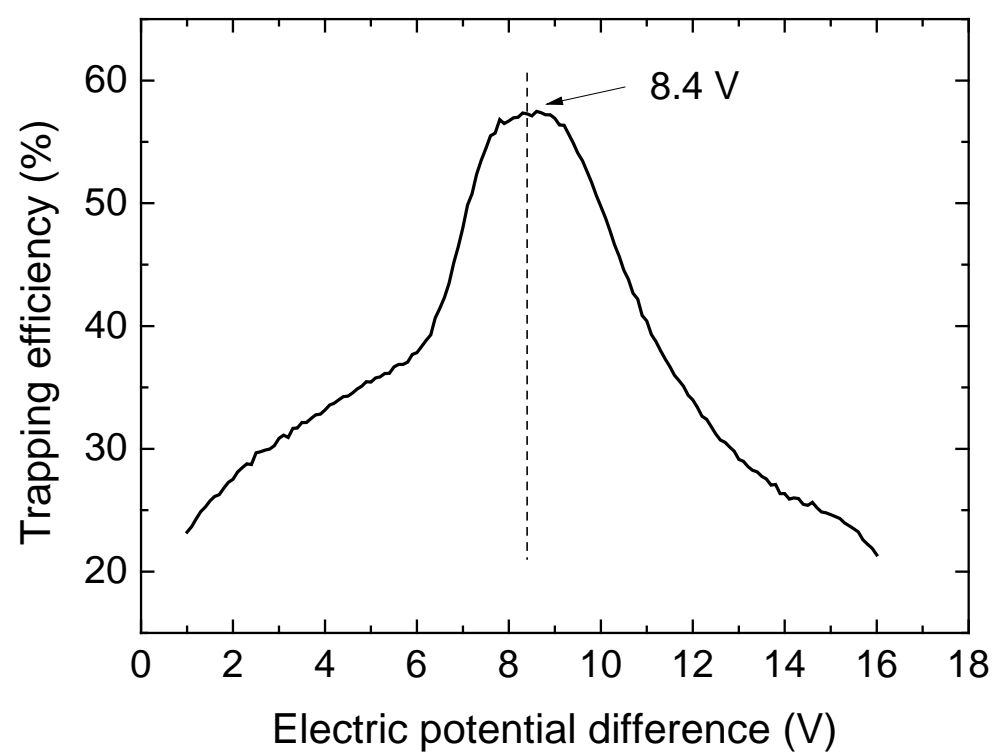

Figure 9. Simulated trapping efficiency in stage I as a function of electric potential difference between the inlet and the stage I electrode.

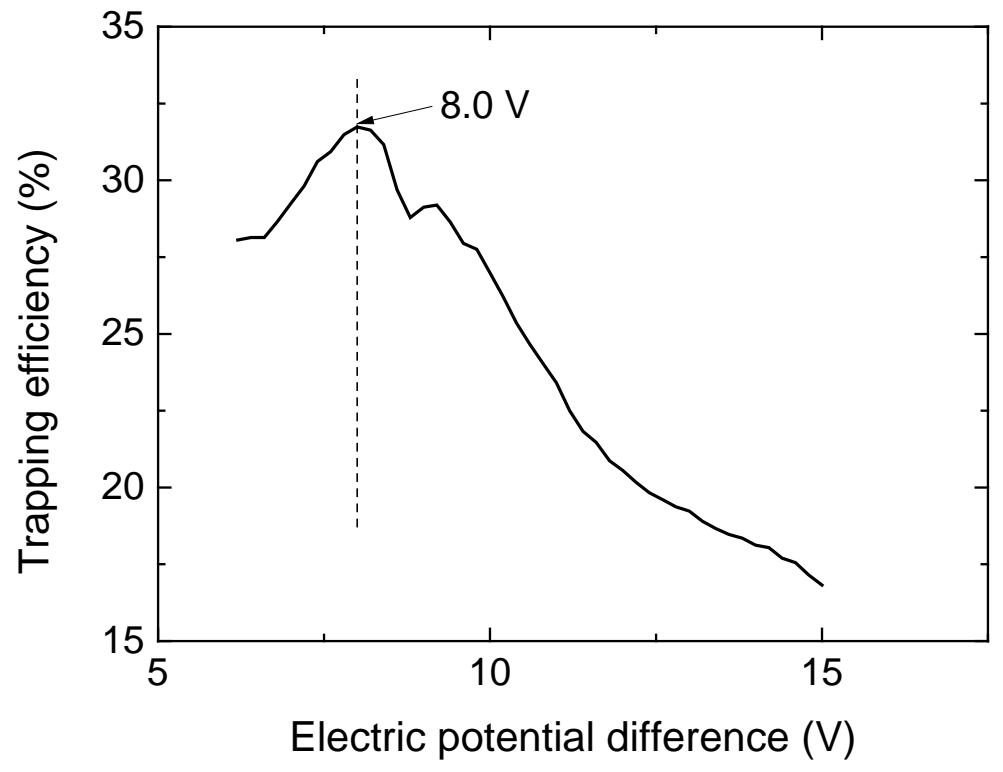

Figure 10. Simulated trapping efficiency in stage II as a function of electric potential difference between the stage I and II electrodes.

\subsection{Simulation of the optimized trap}

Detailed simulation parameters of an optimized three stage trap, based on the previous analysis are given in table 2, while the results of this simulation are presented in figures 11-15. Figure 11 shows trapping efficiency and loss processes over time. Trapping efficiency is increased significantly compared to the values obtained before optimization, and achieved efficiency of $17 \%$ is in line with readily reported efficiencies of $10-20 \%$ in empirically optimized experimental traps that use similar potentials and other parameters [57]. Still, positronium formation represents the most prominent loss process removing $75 \%$ of 
positrons. Unfortunately, in three stage traps, this loss cannot be reduced further as it is governed by the properties of the nitrogen molecule, i.e. its cross-sections for interaction with positrons.

Table 2 Simulation parameters for the optimized buffer gas trap

\begin{tabular}{llll}
\hline Parameters & Stage I & Stage II & Stage III \\
\hline Electrode radius (mm) & 5 & 20 & 20 \\
Length (m) & 0.5 & 0.5 & 0.5 \\
Pressure (Torr) & $3 \times 10^{-3}$ & $10^{-4}$ & $10^{-5}$ \\
Background gas & $\mathrm{N}_{2}$ & $\mathrm{~N}_{2}$ & $\mathrm{~N}_{2}{ }^{0.5}+\mathrm{CF}_{4}{ }^{0.5}$ \\
Magnetic field (G) & 530 & 530 & 530 \\
Electrode potential (V) & 16 & 8 & 0 \\
\hline \multicolumn{5}{l}{} \\
\hline Inlet electrode potential (V) & Initial conditions & \\
$\mathrm{e}^{+}$source bias (V) & & 24.4 & \\
Energy distribution width (FWHM) (eV) & 0.1 & \\
\hline
\end{tabular}

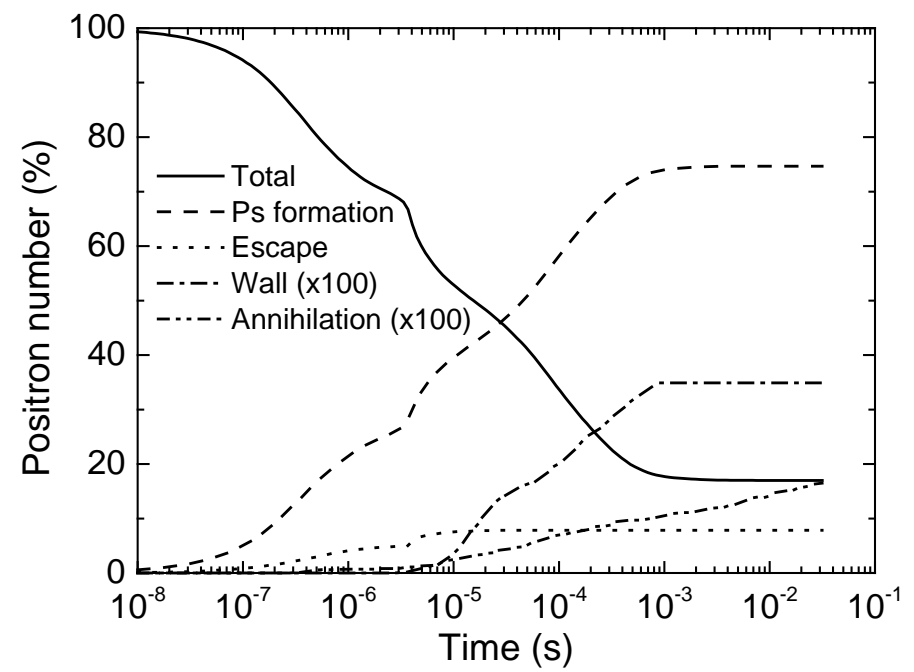

Figure 11. Total number of remaining positrons and the individual loss processes over time, normalized to the number of the initial positrons for the optimized trap. Losses to walls and direct annihilation have been multiplied by a factor of 100 for visibility

Figure 11 also shows that, despite the optimization of pressure, a number of particles (8\%) still escape the trap. To explain why this loss cannot be further reduced by pressure optimization (for the given initial beam properties) we can look at figure 11 showing the relative number of positrons in individual stage over time, with escape loss process overlaid. It is apparent that a small number of positrons manage to escape the trap even before any of the positrons enter stages II or III. Those positrons are not reflected by the outlet electrode potential, but are backscattered by collisions with $\mathrm{N}_{2}$ molecule inside stage I and, still having enough energy to travel over the inlet electrode potential, leave the simulation. A small, but sudden, increase is noticeable in the curve that depicts the positron escape, marked by arrow, which represents the moment when the bulk of positrons, that were reflected by the outlet electrode potential, reach the inlet and leave the trap. So the positrons lost before that moment are lost through backscattering 
on $\mathrm{N}_{2}$ molecules, and positrons lost after that moment are those that made the trip through the trap and back without losing enough energy. The latter group can be reduced by further increasing the gas pressure, but that, on the other hand, increases the amount of backscattering. Therefore, the $8 \%$ loss is the optimized minimal value obtainable for given incoming beam properties at the stage I with pressure of 3 mTorr.

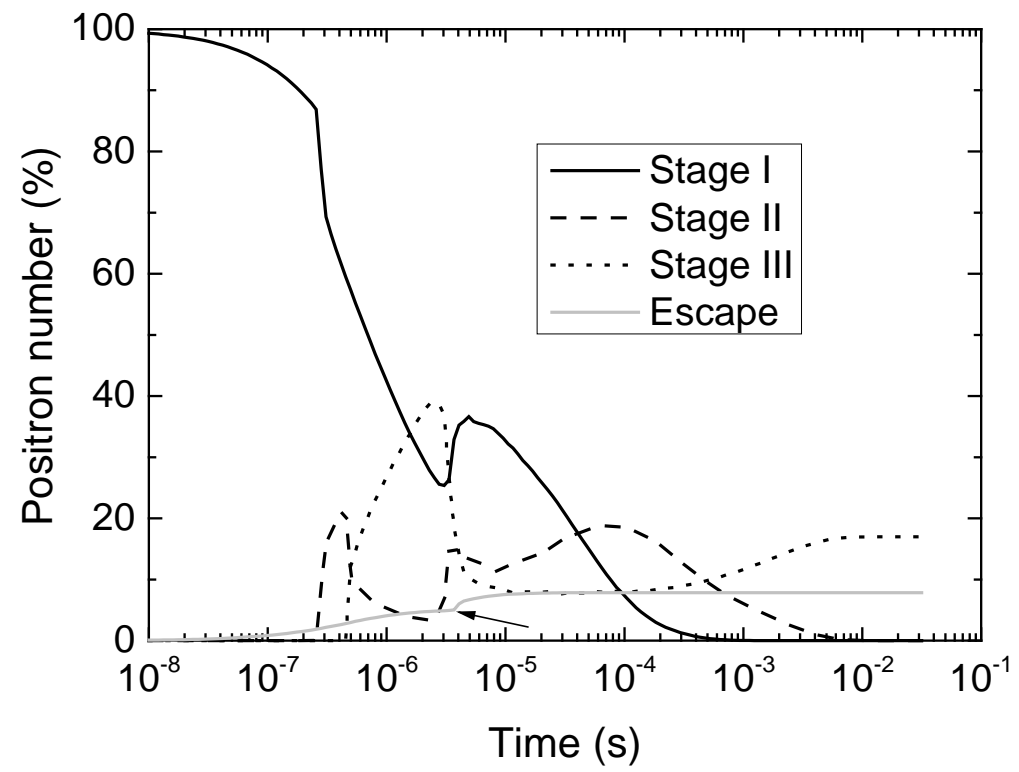

Figure 12. Relative number of positrons in individual stages over time in the optimized trap. Light line represents the amount of positrons escaped over time.

Mean kinetic energy of positrons inside individual stages as well as the mean kinetic energy of the complete swarm (total) over time is given in figure 13. Positrons entering the second stage at $\sim 3 \times 10^{-7}$ are accelerated by the voltage drop as well as those entering the stage III, making maximum kinetic energy in respective stages 19 and $27 \mathrm{eV}$. Peak of the kinetic energy occurring in stage I is a result of higher energy positrons entering the stage I again after reflection off the outlet electrode without experiencing a single electron excitation, a collision resulting in a significant energy loss. Gradually all of the positrons are accumulated in the third stage and after $\sim 3$ ms mean energy of positrons in stage III practically becomes the energy of the entire swarm even though a small number of positrons remains in stage II. By $40 \mathrm{~ms}$ the entire swarm is thermalized in the third stage through vibrational excitation of $\mathrm{CF}_{4}$ and rotational excitation of $\mathrm{N}_{2}$. 


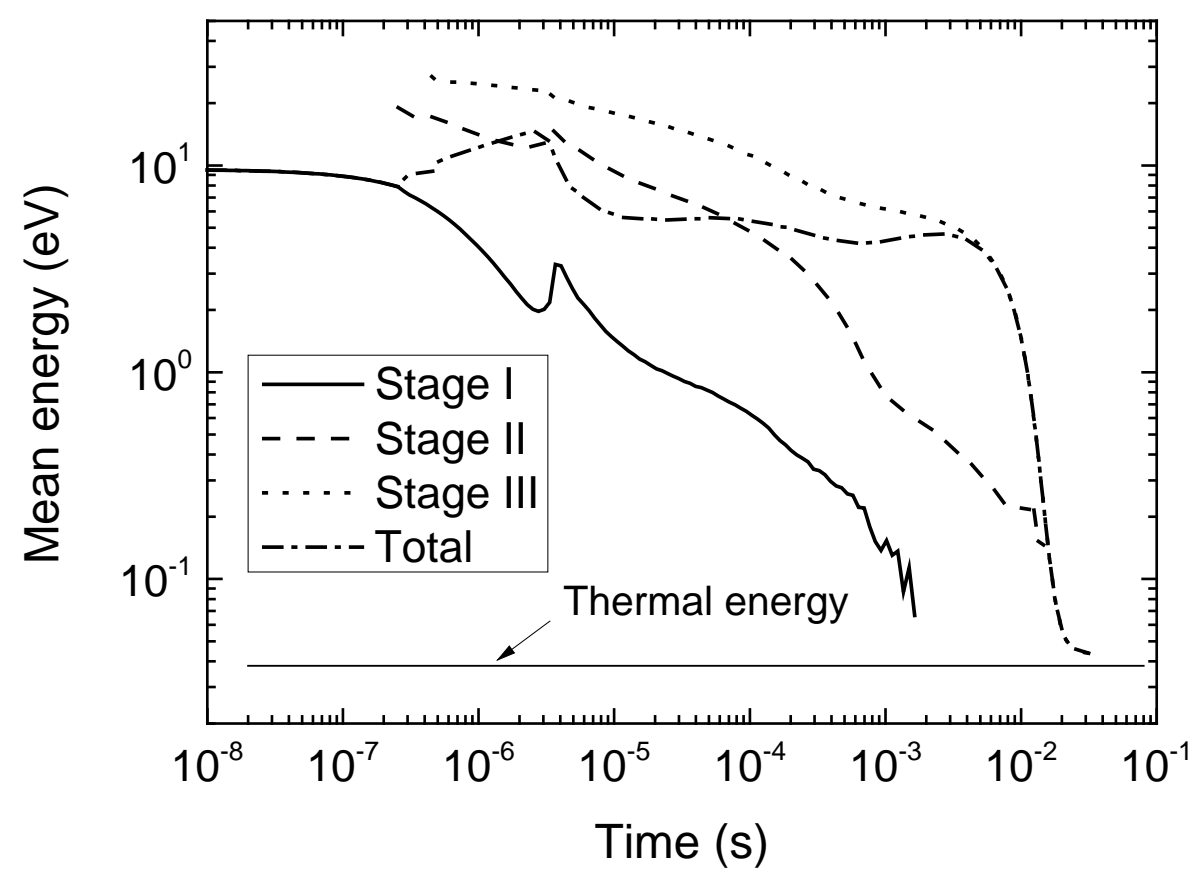

Figure 13. Simulated mean kinetic energy of positrons in individual stages and mean kinetic energy of the entire positron swarm inside the optimized trap, over time.

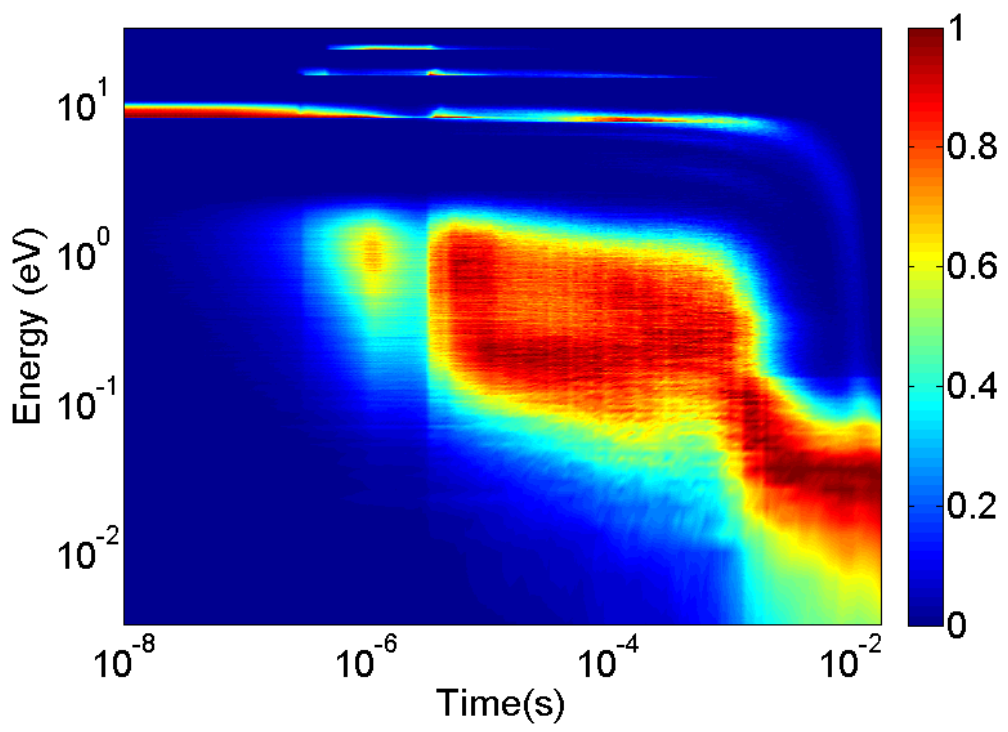

Figure 14. Evolution of positron energy distribution during thermalization inside a optimized buffer gas positron trap.

Evolution of kinetic energy distribution of positrons for the optimized trap is presented in figure 14, sampled in the same manner as figure 3. Again, it can be seen how the initial positron beam splits into several distinct ensembles as a result of stage to stage acceleration and discreet energy losses through electron excitation of $\mathrm{N}_{2}$, and their final thermalization to a Maxwellian distribution. Compared to notoptimized circumstances in figure 3, figure 13 shows a cleaner situation in a sense that, due to the fact that 
the electrode voltages are well tuned to the energy loss of $a^{1} \Pi$ excitation only four distinct ensembles (in respect to their energy) can be discerned, with better defined energies. Still the lowest energy ensemble has a very wide distribution, that is not similar to the Maxwellian (figure 15) necessitating the use of full kinetic and non-hydrodynamic swarm approach.
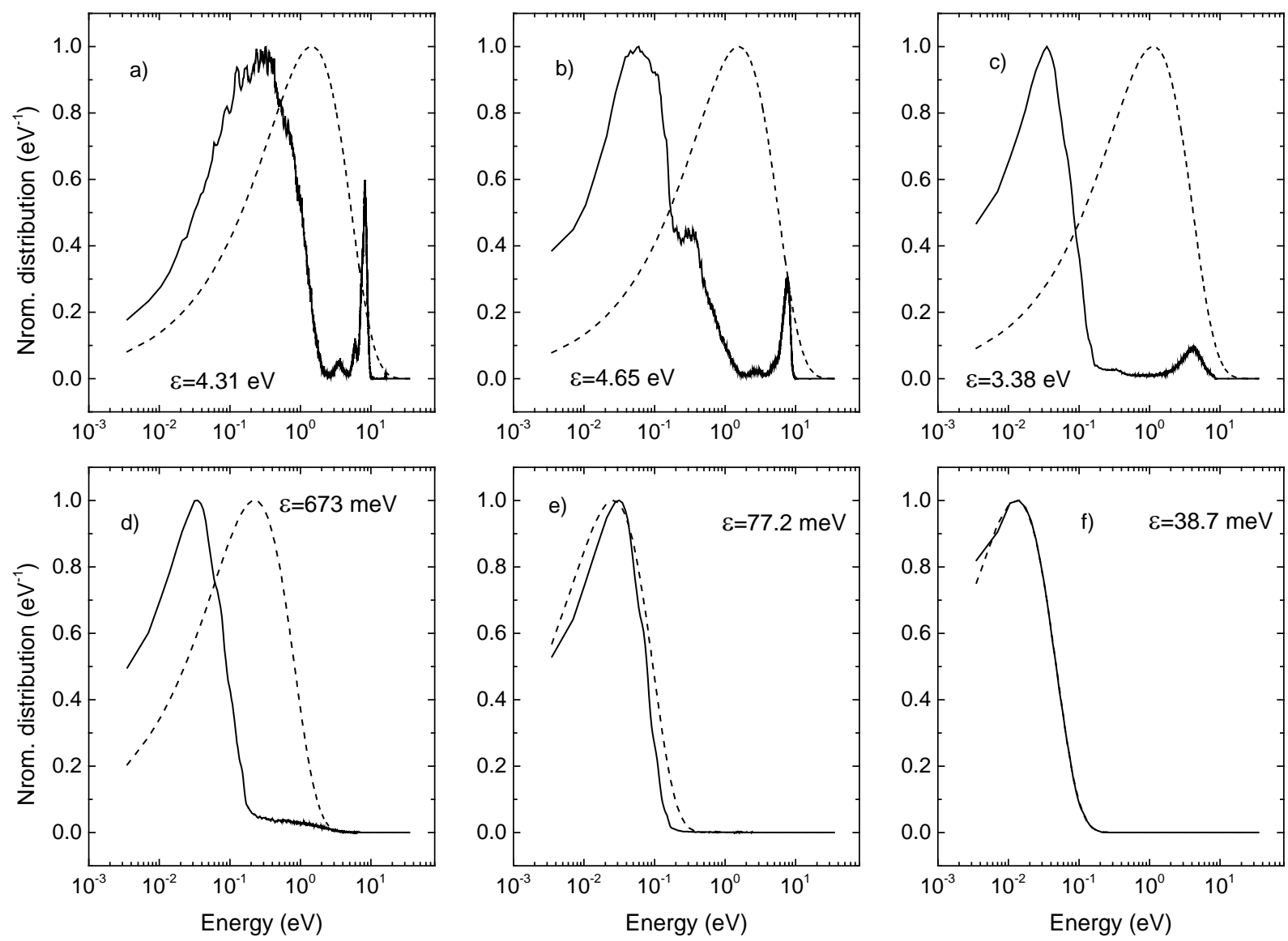

Figure 15. Evolution of positron energy distribution during the final stage of thermalization in accumulation region at moments a) $0.84 \mathrm{~ms}$, b) $1.99 \mathrm{~ms}$, c) $6.24 \mathrm{~ms}$, d) $12.15 \mathrm{~ms}$, e) $17.79 \mathrm{~ms}$, f) $256.62 \mathrm{~ms}$. Dashed lines represent MB distribution for the appropriate mean energy.

\subsection{Simulation of a two stage trap}

From previous results, it is clear that the Ps formation is the main limiting factor for trapping efficiency, limiting it to $57 \%$ per stage. However, reducing the voltage drop in the third stage to a far smaller value, like $1 \mathrm{~V}$, that is big enough to axially contain a thermalized positron swarm on one hand, and small enough, on the other, not to induce another round of electron excitations and Ps formation, could provide a significant increase in overall efficiency. Positrons can lose energy, and stay confined in this accumulation region, through vibrational excitation of $\mathrm{CF}_{4}$ and rotational excitation of $\mathrm{N}_{2}$ molecules. The issue becomes, whether the pressure in the third stage (or stage IIa) is sufficient to provide efficient thermalization of positrons into the accumulation region before positrons are lost in the second stage to processes of direct annihilation and diffusion to the walls due to the fact that positrons remain in higher pressure region (that corresponding to the second (II) stage) for longer times. Figure 16 illustrates the electric potential structure of the described modification, while the rest of simulation parameters correspond to the table 2 . 
Simulation results for the described two stage design are shown in figures 17-21. Figure 17 shows the loss processes over time, and the relative number of positrons remaining in the trap (total). Number of escaping positrons does not differ from the optimized case, as it is mostly determined by the pressure of nitrogen in stage I and the settings of inlet and stage I electrode with respect to the energy of the incoming positron beam. Increase of the total efficiency is achieved by reducing the overall number of Ps formation events below $60 \%$ by eliminating them from the stage IIa (effectively the third stage without a large potential drop). Overall trapping efficiency achieved is 33\%. Positron loss through direct annihilation and diffusion to walls is larger than in previous simulations as expected (curves that correspond to these processes have been scaled by a factor of 10 in figure 17). This is due to the fact that positrons spend more time in the stage II where the pressure is higher. However, both processes do not amount to losses by more than a couple percent so one does not need to further reduce the pressure in the stage IIa.

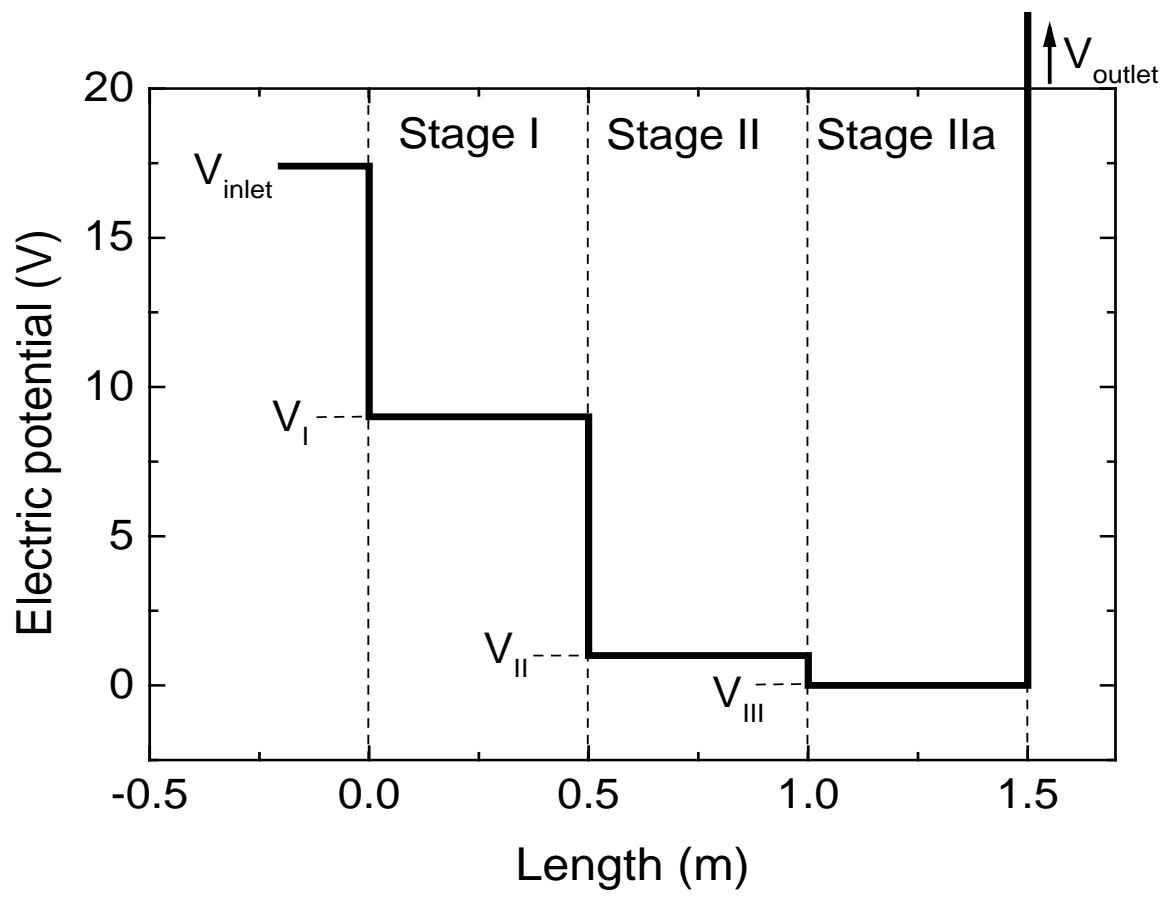

Figure 16. Electric potential profile of a two-stage design 


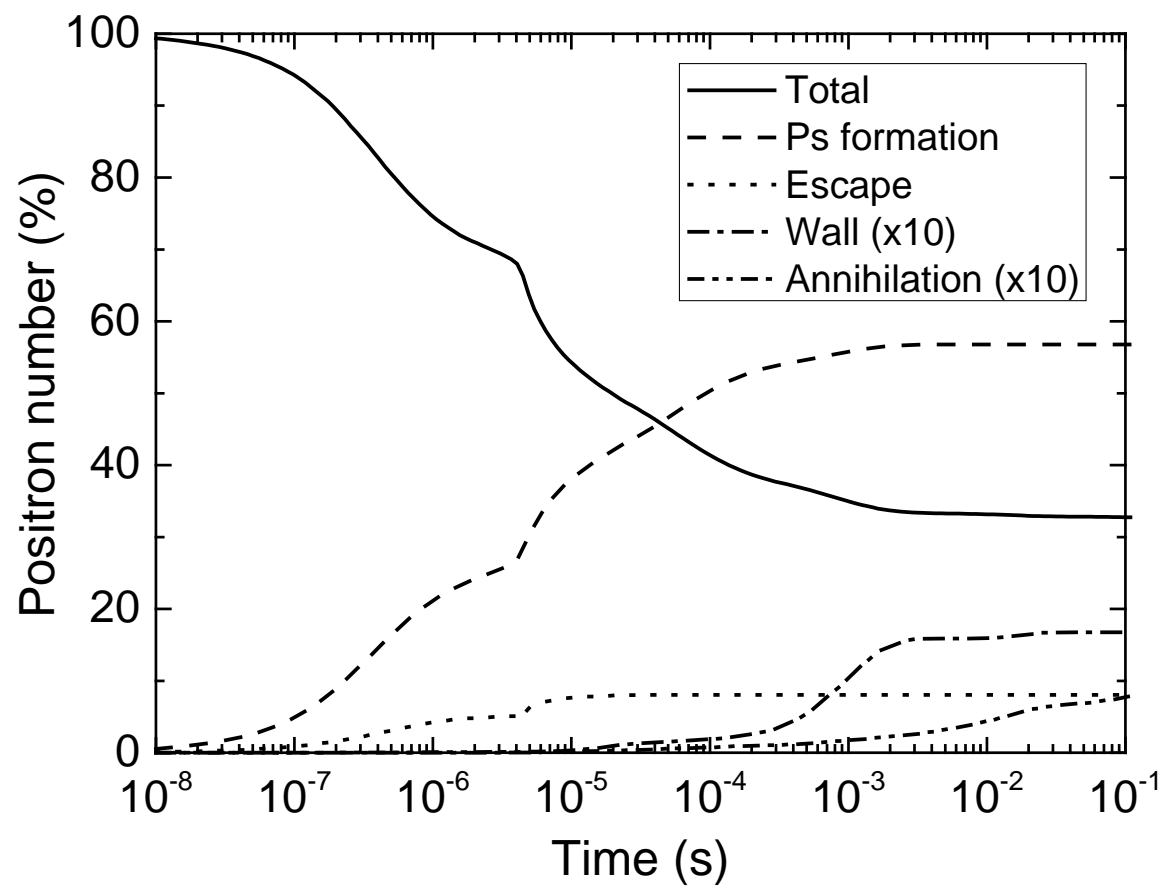

Figure 17. Total number of remaining positrons and the individual loss processes over time, normalized to the number of initial positrons for the two stage trap simulation. Losses to the walls and direct annihilation have been multiplied by a factor of 10 for visibility

Positron thermailzation is still achieved in the same timescale (figure 18) but positrons stay in stage II longer, as the stage IIa filling dynamic is significantly changed compared to the three stage traps (with respect to potential stages) as shown in figures 12 and 19. The reason for this is a significant difference in nature and scale of cross-sections for $a^{1} \Pi$ electron excitation of $\mathrm{N}_{2}$ and vibrational excitations of $\mathrm{CF}_{4}$ molecules which is the most significant process at energies of few eV. Energy loss in one electro excitation of $\mathrm{N}_{2}$ is $\sim 8.5 \mathrm{eV}$ while the energy loss in one vibrational excitation of $\mathrm{CF}_{4}$ is $\sim 150 \mathrm{meV}$. Apparently, while a single electron excitation collision is sufficient to confine a positron to a certain stage, in the case of a two stage trap, it takes tens of vibrational collisions for a positron to lose enough energy and remain trapped in the last stage (figure 20). 


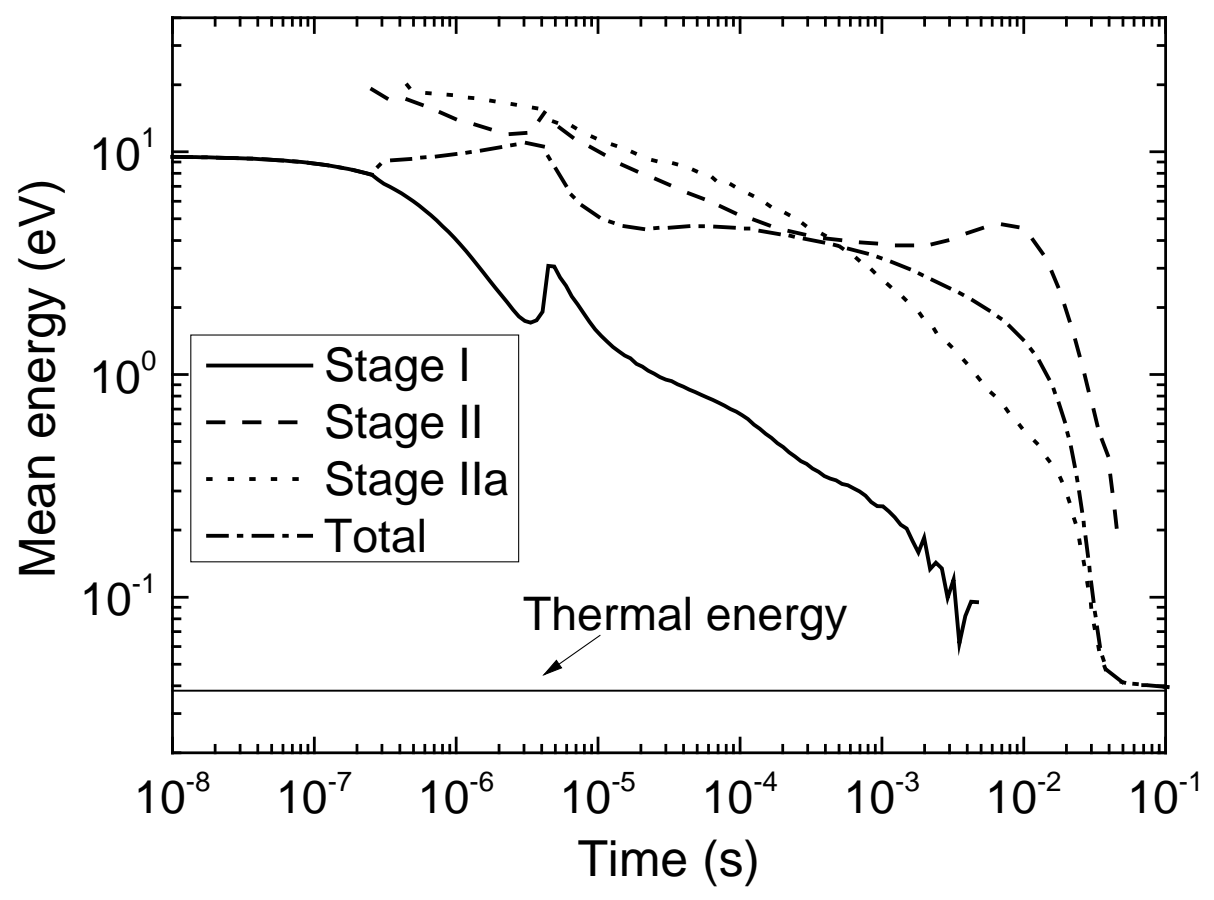

Figure 18. Simulated mean kinetic energy of positrons in individual stages and mean kinetic energy of the entire positron swarm inside the two stage trap, over time.

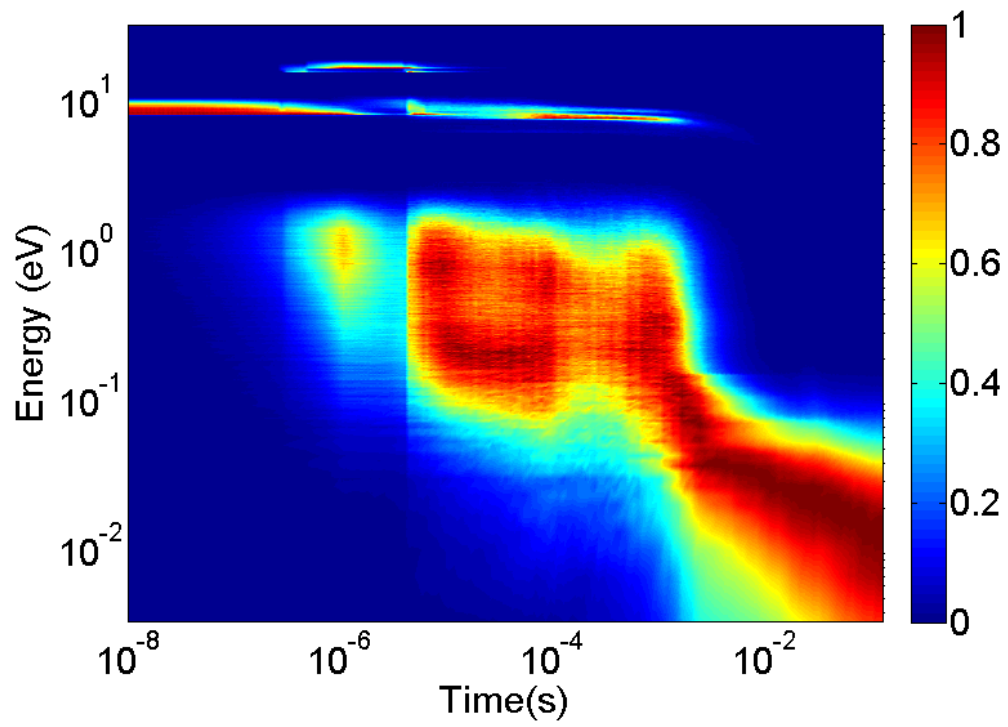

Figure 19. Evolution of positron energy distribution during thermalization inside a two stage buffer gas positron trap. 

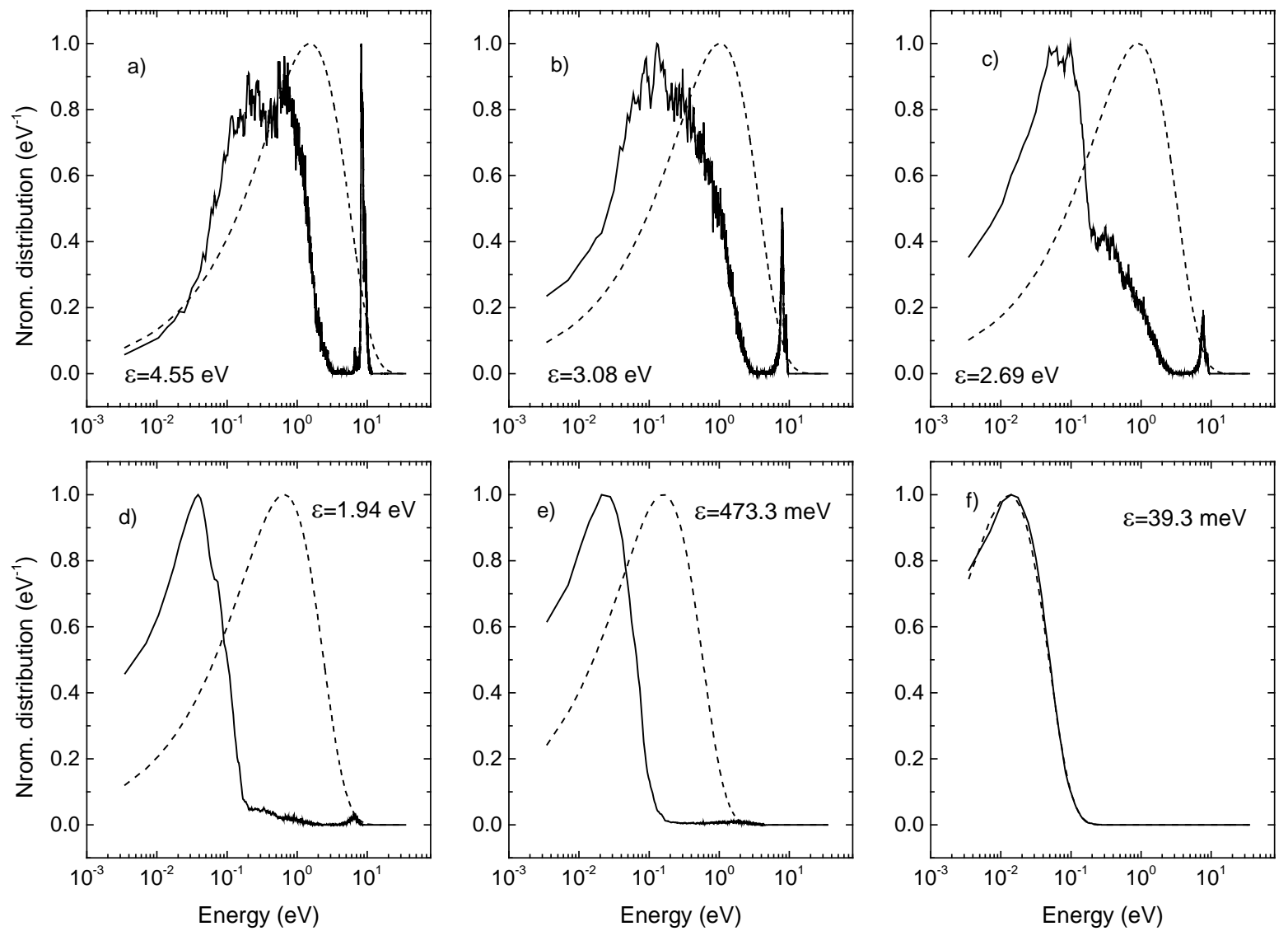

Figure xx. Evolution of positron energy distribution during the final stage of thermalization in accumulation region at moments a) $0.09 \mathrm{~ms}$, b) $1.36 \mathrm{~ms}$, c) $2.24 \mathrm{~ms}$, d) $5.7 \mathrm{~ms}$, e) $21.53 \mathrm{~ms}$, f) $131.69 \mathrm{~ms}$. Dashed lines represent MB distribution for the appropriate mean energy.

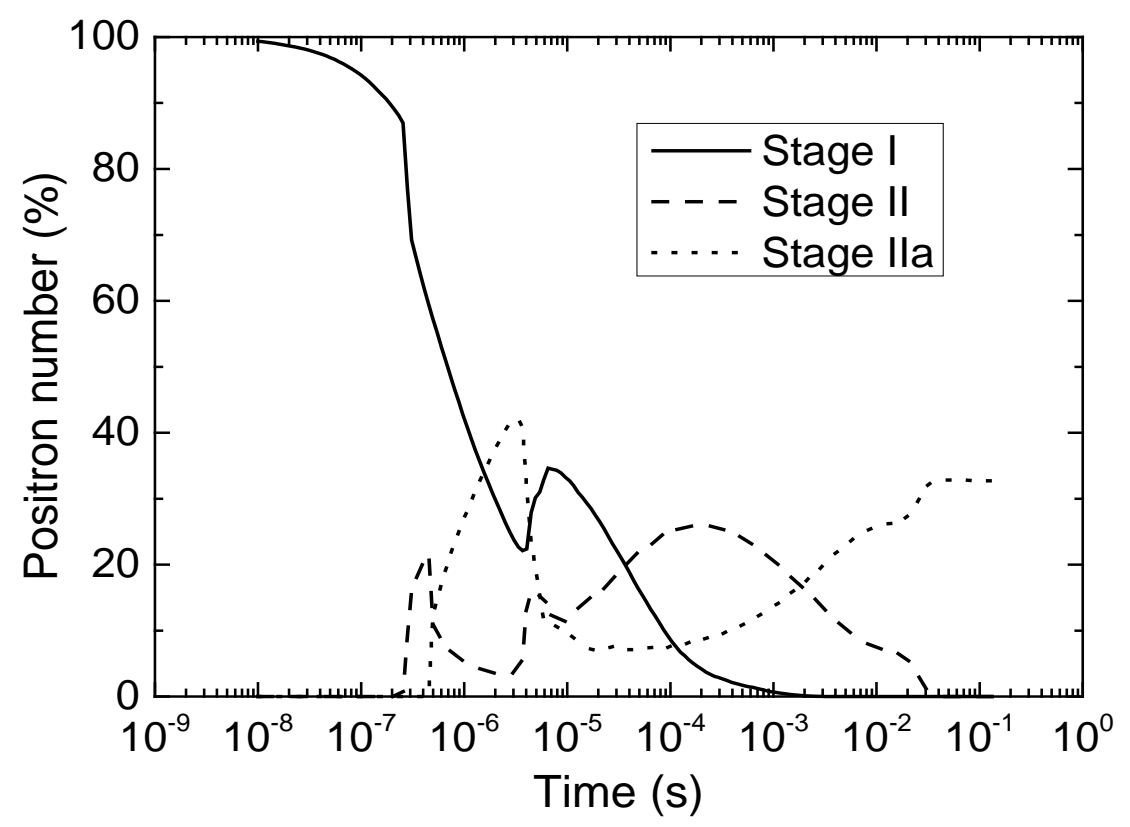


Figure 20. Relative number of positrons in individual stages over time in the two stage trap.

The fact that positrons, in the case of a two stage trap, remain in the second stage, where $\mathrm{N}_{2}$ pressure is 0.1 mTorr, up to six times longer leads to somewhat wider spatial distribution at the end of thermalizaton which can be seen on figure 21. This provides an indication as to why this approach is not used in practice as much as its increased efficiency would warrant. Similar approach has been attempted by Murphy and Surko [5] by testing trapping efficiency in a so called "shallow well" regime that used the same principle as described in this subsection, albeit with the potential well depth in stage IIa of $4 \mathrm{~V}$. They arrived to the conclusion that the trap in this regime is much more sensitive to any misalignments of electric and magnetic field (which are collinear ideally). In case there is a small angle between electric and magnetic field vectors in the small area between electrodes, where electric field exists, during a single crossing of a single positron, its trajectory does not deviate significantly from the ideal case (which is assumed in this simulation). However, as in the two stage variant, the accumulation stage is filling up much slower, it is a given that positrons will cross the electrode boundary a large number of times. During each crossing, the small angle between the electric and magnetic field induces a small drift that might lead to transport of positrons to electrode walls. Our code, at this point, is not capable of simulating these imperfections due to misalignment, and therefore these losses are not included in the simulation.

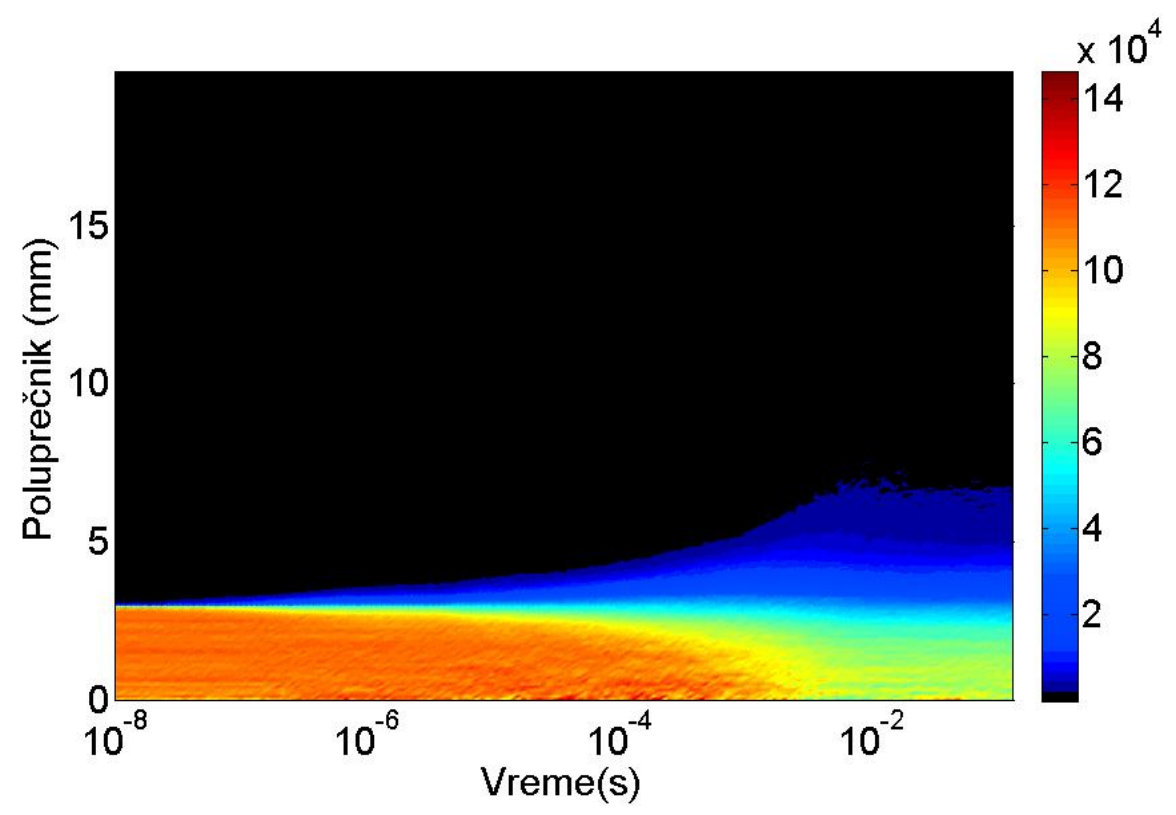

Figure 21. Evolution of spatial distribution of the positron swarm during thermalization in a two stage trap.

\section{Conclusion}

This paper presents a numerical Monte Carlo simulation of positron buffer gas operation, following positrons from the moment they enter the trap, to the moment they thermalize. As the positrons travel through the trap, the initial well defined beam, due to inelastic collisions and acceleration by electrode potential drops, differentiates into several ensembles of positrons, some of which have relatively wide energy distributions. This type of particle behaviour prompts the use of full kinetic treatment within the, so called, swarm methods in a quantitative analysis of trap operation. Eventually, all of the positrons thermalize in the final, accumulation stage and the thermalization time is mostly determined by the pressure and composition of buffer gas in the accumulation region. All salient features of the gas filled traps have been explained earlier by mostly beam like arguments [5] and recently also by using Maxwellian distribution function at all times during the last stages of the thermalization [22]. However, 
for quantitative description, a swarm method is necessary both to describe the collapse of the early mono energetic beam(s) through thermalization of the complex distribution of the resulting ensembles into eventual Maxwellians and room temperature defined mean energy. This can be clearly seen from out presentation of the distribution functions. We also present averaged properties useful in quantifying the performance of the gas filled traps such as trapping efficiency and also to perform optimizations of the trap parameters. In principle such averaged properties may be used in the lack of measured data for the well defined swarm transport coefficients to fix the cross sections. Certainly the thermalization time would define the energy exchange processes at high, moderate and mostly low energies. However. before doing that we need to define the conditions for measurements in traps. Approach to thermal mean energy is asymptotic, i.e. it takes forever and in the past thermalization times both in experiments and in theory were defined as breaking through the levels $50 \%$ or $10 \%$ above the thermal energy both of which may be established more precisely. The second requirement would be to define better the collision processes used, their superelastic counterparts and energy losses.

Using our trap model, we were able to optimize gas pressure and electrode potentials to achieve best possible operation for a given electrode geometry and incoming positron beam properties. By analyzing loss processes we were able to identify the contribution of backscattering which has not been explicitly discussed before, although implicitly it is noticed in optimization of gas pressure. The number of backscattered positrons, derived in this paper should be taken with caution as we did not include the differential scattering data (that became available recently [58]). The main loss process, as expected, is shown to be Ps formation on nitrogen molecules. Due to the width of positron energy distribution coming from a solid Ne moderator, as a rule of a thumb, $\sim 40 \%$ of positrons are lost every time a positron has an opportunity to excite electron levels of $\mathrm{N}_{2}$. In that respect, even though the simulated trap geometry and gas pressures are more in line with devices that are commonly referred to as two-stage traps, we still discuss three stages, that are determined by applied electrode potentials, as it is the potentials that govern what types of events are available to positrons.

Achieved optimal trapping efficiency (for given simulated conditions) of $18 \%$ is in line with readily reported efficiencies of $10-20 \%$ (for similar potential drops and other properties)[57]. We were not, however, able to reach maximum reported efficiency of $30 \%$ [57] mainly due to the relatively simple description of electric potential. These high performance traps have more complex electrode and potential structures that can form gradients in potential, leading to less Ps formation and better trapping performance.

In order to reduce Ps formation losses, a simulation of a two-stage trap (two stages in regards to electric potential) has been performed. Removal of the third $8 \mathrm{~V}$ potential drop significantly reduced the amount of Ps formation, increasing the trapping efficiency to $33 \%$. This however is not in line with the findings of Murphy and Surko [5], who described this potential shape as shallow well regime. They did however discover that poor trap performance in this regime can be somewhat improved by better field alignment. The simulation suggests that the diffusion to walls or direct annihilation, even though the particles spend more time in the higher pressure region, still do not contribute to losses significantly. The plausible cause for disagreement between simulation and experimental findings is the fact that our simulation assumes ideally axial magnetic field, as well as ideally axial and infinitely short electric field between electrodes. Due to the fact that positrons a in shallow well regime fill accumulation stage much slower, causing them to repeatedly cross the interface between the second and accumulation stage, misalignment in electric and magnetic fields induces radial drift that ultimately might lead to positron losses. Simulation of such imperfect fields will be the subject of our future work. However, we do feel that further experimental work on this effect is merited, as suppression of this loss could lead to improved trapping efficiency, either through the method described in this paper, or through other trapping schemes.

We hope that or results provide evidence that quite accurate representation of the gas filled traps may be allowed by using the same tools as in physics of ionized gases (swarms, discharges, low 
temperature and collisional plasmas, etc.). In addition to quantitative comparison the simulation opens the door to definition and identification of different kinetic phenomena.

References:

[1] Canter K F, Coleman P G, Griffith T C and Heyland G R 1972 J. Phys. B: At. Mol. Phys. 5 L167

[2] Shultz P J and Lynn K G 1988 Rev. Mod. Phys. 60701

[3] P G Coleman 2000 Positron Beams and Their Applications ed P G Coleman (Singapore: World Scientific)

[4] Surko C M, Leventhal M and Passner A 1989 Phys. Rev. Lett. 62901.

[5] Murphy T J and Surko C M 1992 Phys. Rev. A 465696.

[6] Charlton M and Humberston J 2000 Positron Physics (New York: Cambridge University Press)

[7] Mills Jr A P and Gullikson E M 1986 Appl. Phys. Lett. 491121

[8] Surko C M, Gilbert S J and Greaves R G 1999 Progress in creating low-energy positron plasmas and beams, in Non-Neutral Plasma Physics, ed J J Bollinger, R L Spencer and R C Davidson (American Institute of Physics, New York)

[9] Fitzakerley D W, et al. 2013 Bull. Am. Phys. Soc. 58 , 176

[10] Marler J P and Surko C M 2005 Phys. Rev. A 72062713

[11] Marler J P and Surko C M 2005 Phys. Rev. A 72062702

[12] Marler J P, Sullivan J P and Surko C M 2005 Phys. Rev. A 71022701

[13] Makochekanwa C, Bankovic A, Tattersall W, Jones A, Caradonna P, Slaughter D S, Nixon K, Brunger M J, Petrovic Z, Sullivan J P and Buckman S J 2009 New J. Phys. 11103036

[14] Cassidy D B, Hisakado T H, Tom H W K and Mills Jr A P 2012 Phys. Rev. Lett. 108133402

[15] Andresen G B et al. 2010 Nature 468673

[16] Amoretti M et al. 2002 Nature 419456

[17] Gabrielse G et al. 2002 Phys. Rev. Lett. 89213401

[18] Cassidy D B, Crivelli P, Hisakado T H, Liszkay L, Meligne V E, Perez P, Tom H W K and Mills Jr A P 2010 Phys. Rev. A 81012715

[19] Sullivan J P, Roberts J, Weed R W, Went M R, Newman D S and Buckman S J 2010 Meas. Sci. Technol. 21085702

[20] Charlton M 2009 J. Phys.: Conf. Ser. 162012003

[21] Al-Qaradawi I, Charlton M, Borozan I and Whitehead R 2000 J. Phys. B: At. Mol. Opt. Phys. 33 2725

[22] Natisin M R, Danielson J R and Surko C M 2014 J. Phys. B: At. Mol. Opt. Phys. 47225209

[23] Shizgal B, Ness K F 1987 J. Phys. B: Atom. Molec. Phys. 20847

[24] Robson R E 1986 J. Chem. Phys. 854486. 
[25] Banković A, Marler J P, Šuvakov M, Malović G and Petrović Z Lj 2008 Nucl. Instr. and Meth. B 266 462

[26] Šuvakov M, Petrović Z Lj, Marler J P, Buckman S J, Robson R E and Malović G 2008 New J. Phys. 10053034

[27] Banković A, Dujko S, White R D, Marler J P, Buckman S J, Marjanović S, Malović G, García G and Petrović Z Lj 2012 New J. Phys. 14035003

[28] Banković A, Dujko S, White R D, Buckman S J and Petrović Z Lj 2012 Nucl. Instrum. Meth. B 279 92-5

[29] Bankovic A, Petrovic Z Lj, Robson R E, Marler J P, Dujko S and Malovic G 2009 Nucl. Instrum. Meth. B 267350

[30] Garcia G, Petrovıc Z L, White R and Buckman S 2011 IEEE Trans. Plasma Sci. 392962

[31] Fuss M C, Sanz A G, Munoz A, Blanco F, Brunger M J, Buckman S J, Limo-Vieira P and Garcia G 2014 Appl. Rad. Isotop. 83159

[32] Petrović Z Lj, Marjanović S, Dujko S, Banković A, Malović G, Buckman S, Garcia G, White R and Brunger M 2014 Appl. Rad. Isotop. 83148

[33] Marjanović S, Banković A, White R D, Buckman S J, Garcia G, Malović G, Dujko S and Petrović Z Lj 2015 Plasma Sources Sci. Technol. 24025016

[34] Blanco F, Roldán A M, Krupa K, McEachran R P, White R D, Marjanović S, Petrović Z Lj, Brunger M J, Machacek J R, Buckman S J, Sullivan J P, Chiari L, Limão-Vieira P and García G 2016 J. Phys. B: At. Mol. Opt. Phys. 49145001

[35] Cassidy D B, Deng S H M, Greaves R G and Mills A P 2006 Rev. Sci. Instrum. 77073106

[36] Clarke J, van der Werf D P, Griffiths B, Beddows D C S, Charlton M, Telle H H and Watkeys P R 2006 Rev. Sci. Instrum. 77063302

[37] Sullivan J P, Jones A, Caradonna P, Makochekanwa C, and Buckman S J 2008 Rev. Sci. Instrum. 79 113105

[38] Cooper B S, Alonso A M, Deller A, Wall T E and Cassidy D B 2015 Rev. Sci. Instrum. 86103101

[39] Greaves R G and Moxom J 2003 AIP Conf. Proc. 692140

[40] Isaac C A, Baker C J, Mortensen T, van der Werf D P and Charlton M. 2011 Phys. Rev. Lett. 107 033201.

[41] Danielson J R, Dubin D H E, Greaves R G and Surko C M 2015 Rev. Mod. Phys. 87 247-306

[42] Surko C M and Greaves R G 2003 Radiat. Phys. Chem. 68419

[43] Lucas J and Saelee H T 1975 J Phys. D: Appl. Phys. 8640

[44] Reid I D 1979 Aust. J. Phys. 32231

[45] Ness K F, Robson R E 1986 Transport Theor. Stat. Phys. 14257

[46] Raspopović Z M, Sakadžić S, Bzenić S and Petrović Z Lj 1999 IEEE Trans. Plasma Sci. 271241

[47] Petrovic Z Lj, Bankovic A, Dujko S, Marjanovic S, Malovic G, Sullivan J P and Buckman S J 2013 AIP Conf. Proc. 1545115

[48] Marjanovic S, Šuvakov M, Bankovic A, Savić M, Malović G, Buckman S J and Petrović Z Lj 2011 IEEE T. Plasma Sci. 392614 
[49] Banković A, Dujko S, Marjanović S, White R D and Petrović Z Lj 2014 Eur. Phys. J. D 68127

[50] Raspopovic Z M, Sakadžic S, Bzenic S and Petrovic Z Lj 1999 IEEE Trans. Plasma Sci. 271241

[51] Petrovic Z Lj, Raspopovic Z M, Dujko S and Makabe T 2002 Appl. Surf. Sci. 1921

[52] Dujko S, Raspopovic Z M and Petrovic Z Lj 2005 J. Phys. D: Appl. Phys. 382952

[53] Ristivojevic Z and Petrovic Z Lj 2012 Plasma Sources Sci. Technol. 21035001

[54] Dujko S, White R D, Petrović Z Lj and Robson R E 2010 Phys. Rev. E 81046403

[55] Petrovic Z Lj, Dujko S, Maric D, Malovic G, Nikitovic Ž, Šašic O, Jovanovic J, Stojanovic V and Radmilovic-Radenovic M 2009 J. Phys. D: Appl. Phys. 42194002

[56] Petrović Z Lj, Simonović I, Marjanović S, Bošnjaković D, Marić D, Malović G and Dujko S 2016 submitted to Plasma Phys. Control. Fusion

[57] Surko C M and Greaves R G 2004 Phys. Plasmas 112333

[58] de Lucio O G and DuBois R D 2016 Phys. Rev. A 93032710 